\title{
ON ALMOST FINITELY GENERATED NILPOTENT GROUPS
}

\author{
PETER HILTON \\ Department of Mathematical Sciences \\ State University of New York \\ Binghamton, NY 13902-6000 USA \\ and Department of Mathematics \\ University of Central Florida \\ Orlando, FL 32816-6990 USA \\ and \\ ROBERT MILITELLO \\ Department of Mathematics \\ Rhodes College \\ Memphis, TN 38112-1690 USA
}

(Received October 3, 1994)

\begin{abstract}
A nilpotent group $G$ is $\mathrm{fgp}$ if $G_{p}$ is finitely generated (fg) as a $p$-local group for all primes $p$; it is fg-like if there exists a nilpotent fg group $H$ such that $G_{p} \simeq H_{p}$ for all primes $p$. The fgp nilpotent groups form a (generalized) Serre class; the fg-like nilpotent groups do not. However, for abelian groups, a subgroup of an fg-like group is fg-like, and an extension of an fg-like group by an fg-like group is fg-like These properties persist for nilpotent groups with finite commutator subgroup, but fail in general.
\end{abstract}

1991 AMS SUBJECT CLASSIFICATION CODES: 20F18, 20K24, 55P15

\section{INTRODUCTION}

In earlier papers the authors have studied various aspects of the theory of almost finitely generated nilpotent groups (see, eg, [CH 1,2,3;HM]). If $A$ is an abelian group, we say that $A$ is finitely generated at every prime (fgp) if, for all primes $p, A_{p}$ is a finitely generated (fg) $\mathbb{Z}_{p}$-module. We also say that $A$ is fg-like if there is an fg abelian group $B$ such that $A_{p} \cong B_{p}$, for all $p$. We also say that $A$ is $B$-like Obviously an fg-like abelian group is fgp, but the example $\oplus \mathbb{Z} / p$ shows that the converse is false. In $[\mathrm{CH}]$ the authors effectively characterize the fg-like abelian groups among the fgp abelian groups $A$ for which the torsion subgroup $T A$ is a direct summand; and the story is taken further in [M1]

It is straightforward to generalize the notions $f g p$, $f g$-like to nilpotent groups. The generalization of fg-like is immediate; as to the generalization of fgp, we need the concept of a set of generators of a p-local (nilpotent) group. Thus the set $S \subseteq H$, where $H$ is a $p$-local group generates $H$ as p-local group if $H$ is the smallest $p$-local subgroup of $H$ containing $S$. Equivalently, let $\langle S\rangle$ be the subgroup of $H$ generated by $S$. Then $S$ generates $H$ as $p$-local group if $\langle S\rangle_{p}=H$, and we say that $G$ is fgp if, for all $p, G_{p}$ is finitely generated as $p$-local group

Now it is not difficult to show that the class of fgp nilpotent groups constitutes a Serre class in the sense of [HR] That is, the abelian fgp groups form a Serre class in the usual sense, but the basic axiom is generalized to assert that, for any short exact sequence of nilpotent groups

$$
G^{\prime} \mapsto G \rightarrow G^{\prime \prime},
$$

$G^{\prime}$ and $G^{\prime \prime}$ are fgp if and only if $G$ is fgp. 
In this paper we are principally concerned with this basic axiom and its analogue for fg-like groups The other axioms create no problems in either case, since tensor product, torsion product and homology (in positive dimensions) commute with localization

In fact, the basic axiom fails in one particular case, even for abelian groups. For let $A$ be the subgroup of $\mathbb{Q}$ generated by the rationals $\frac{1}{p}$, all $p$ Then $A$ is $\mathbb{Z}$-like, but the embedding $\mathbb{Z} \subseteq A$ induces a short exact sequence

$$
\mathbb{Z} \mapsto A \rightarrow \underset{p}{\oplus} \mathbb{Z} / p
$$

where the quotient, as already mentioned, is not fg-like However, for abelian groups, the other two assertions of the basic axiom for fg-like groups do hold We may put this in the following way

THEOREM 0.1. In the category of abelian groups

i a subgroup of an fg-like group is fg-like,

ii an extension of an fg-like group by an fg-like group is fg-like

We thus devote much attention to the status of the extension of Theorem 01 to nilpotent groups

The plan of the paper is as follows In Section 1 we prove that the class of fgp nilpotent groups is a Serre class, most of this has appeared already in the literature (see [H2]), but we have felt it desirable to make this paper largely self-contained In Section 2 we give the (easy) proof of Theorem 01 In Section 3 we show that Theorem 01 does extend to an interesting subclass of the class of nilpotent groups, namely, to the class of nilpotent groups $G$ with finite commutator subgroup $[G, G]$ It is interesting to remark that, for fgp nilpotent groups $G$, this class coincides with the class of those $G$ such that $G / Z G$ is finite, where $Z G$ is the center of $G$.

In Section 4 we show that both parts of the analogue of Theorem 0.1 fail for the class of nilpotent groups Indeed, we construct a torsion free nilpotent fg-like group $H$ of rank 2, admitting a subgroup $G$, such that (i) $G$ is not fg-like and (ii) $G$ is an extension of an fg-like abelian group by an fg-like abelian group Although our construction is specific, we point out that the construction method can be applied to produce a continuum of such counterexamples.

We close this introduction by explaining the genesis of the ideas of fgp and fg-like nilpotent groups in homotopy theory Mislin (see [M2]) introduced the idea of the genus of an fg nilpotent group $N$ and that of the genus of a nilpotent space of finite type $X$. Thus the genus of $N$ is the set of isomorphism classes of fg nilpotent groups $M$ such that $N_{p} \cong M_{p}$ for all primes $p$; and the genus of $X$ is the set of homotopy types of nilpotent spaces $Y$ of finite type such that $X_{p} \simeq Y_{p}$ for all primes $p$. We say that $M$ is in the genus of $N$ and that $Y$ is in the genus of $X$, writing $M \in \mathcal{G}(N), Y \in \mathcal{G}(X)$ Plainly if $Y \in \mathcal{G}(X)$, then $\pi_{1} Y \in \mathcal{G}\left(\pi_{1} X\right)$, where $\pi_{1}$ is the fundamental group.

The finiteness restrictions on $N$ and $X$ are designed to render $\mathcal{G}(N)$ and $\mathcal{G}(X)$ calculable - indeed, $\mathcal{G}(N)$ will be finite, and $\mathcal{G}(X)$ will be finite if $X$ is compact. However, there is no reason in principle for these finiteness restrictions. In particular, the finiteness restriction on $N$ renders the notion of genus uninteresting in the case that $N$ is abelian, since then $\mathcal{G}(N)$ is trivial. In [H1] the author initiated a "controlled departure" from this restriction, which was carried further in [CH1], we still require $N$ to be fg but allow $M$ to be arbitrary. This led to the notion of the extended genus; and, of course, the extended genus of $N$ is just the set of isomorphism classes of $N$-like (nilpotent) groups, and the question arises of how to characterize fg-like nilpotent (or abelian) groups in the class of all nilpotent (or abelian) groups.

Since the notion of fg-like is so closely related to localization (at every prime), it was natural to introduce, as we have done in this paper, the idea of groups which are $f g$ at every prime into the attempt to characterize fg-like groups When it turned out that this class of groups satisfied the Serre axioms, we became convinced that it was really worth studying.

Of course, we may similarly define the extended genus of a nilpotent space of finite type A beginning to the study of this notion was made in [H2], where one started with a nilpotent space of finite 
type which was a circle bundle In its extended genus one found nilpotent spaces which were $K(\pi, 1)$ bundles where $\pi$ is a group of pseudo-integers [H1], that is, a group in the extended genus of a cyclic infinite group Of course, just as for the genus, if $Y$ is in the extended genus of $X$, then $\pi_{1} Y$ is in the extended genus of $\pi_{1} X$

Notice that the notions fg-like and fgp are related by "commuting quantifiers" Thus $N$ is fg-like if $\exists M . \forall p . M_{p} \cong N_{p}$, and $N$ is fgp if $\forall p . \exists M . M_{p} \cong N_{p}$ (Here $M$ is, of course, fg nilpotent )

\section{THE SERRE CLASS OF FGP NILPOTENT GROUPS}

Here we review the arguments which show that the class of fgp nilpotent groups forms a Serre class in the extended sense of $[\mathrm{HR}]$, recall that effectively this just means that the abelian fgp groups form a Serre class in the usual sense and that, given the short exact sequence

$$
G^{\prime} \mapsto G \rightarrow G^{\prime \prime}
$$

of nilpotent groups, then $G$ is fgp if and only if $G^{\prime}$ and $G^{\prime \prime}$ are fgp

As pointed out in the Introduction, a nilpotent group $G$ is fgp if and only if, for each prime $p$, there exists an fg nilpotent group $H$ such that $H_{p} \cong G_{p}$, indeed, we may even assume that $H \subseteq G_{p}$ so that $H_{p}=G_{p}$ This makes it obvious that the class of abelian fgp groups is closed under tensor product, torsion product and homology, since these constructions commute with localization and preserve finite generation Indeed, for the same reason, the class of nilpotent fgp groups is closed under homology. We also claim that the basic axıom of a Serre class, relating to (1 1), obviously holds for abelian fgp groups. We now prove the basic axiom in general

THEOREM 1.1. Let $G^{\prime} \mapsto G \rightarrow G^{\prime \prime}$ be a short exact sequence of nilpotent groups. Then $G$ is fgp if and only if $G^{\prime}$ and $G^{\prime \prime}$ are fgp.

We base our proof on two important lemmas

LEMMA 1.2. Let $N \mapsto G \stackrel{k}{\rightarrow} Q$ be a central extension with $G$ nilpotent. Then $G$ is fgp if $N$ and $Q$ are fgp

PROOF. Let $p$ be a fixed but arbitrary prime and let $H, L$ be fg subgroups of $N_{p}, Q_{p}$ such that $H_{p}=N_{p}, L_{p}=Q_{p}$. Of course, $N_{p} \mapsto G_{p} \stackrel{k_{p}}{\rightarrow} Q_{p}$ is central. Let $M$ be an fg subgroup of $G_{p}$ mapping onto $L$ and let $K=\langle H, M\rangle$. We claim that $K_{p}=G_{p}$

To see this, let $x \in G_{p}$. Then there exists a $p^{\prime}$-number $q$ such that $k_{p} x^{q} \in L$ so that $k_{p} x^{q}=k_{p} y$, for some $y \in M$, and $x^{q}=y z$, with $z \in N_{p}$ Then there exists a $p^{\prime}$-number $r$ such that $z^{r} \in H$, whence $x^{g r}=(y z)^{r}=y^{r} z^{r} \in K$. This shows that $x \in K_{p}$ and completes the proof of the lemma

REMARK. Of course, the converse of Lemma 1.2 also holds - indeed, it follows from Theorem 11. However, we do not need the converse to prove Theorem 1.1.

Our second lemma in fact exploits Lemma 1.2

LEMMA 1.3. Let $G$ be nilpotent. Then $G$ is fgp if and only if $G_{a b}$ is fgp.

PROOF. We already know that $G_{a b}$ is fgp if $G$ is fgp, since $G_{a b}=H_{1}(G)$. Suppose, conversely, that $G_{a b}$ is fgp. We consider the Hall commutator map ${ }^{1}$

$$
\Phi: \otimes^{2} G_{a b} \rightarrow \Gamma^{2-1}(G) / \Gamma^{2}(G) .
$$

Now $\otimes^{2} G_{a b}$ is fgp, so, by the known facts for abelian groups, $\Gamma^{2-1}(G) / \Gamma^{2}(G)$ is fgp.

Consider now the central extension

$$
\Gamma^{2-1}(G) / \Gamma^{2}(G) \mapsto G / \Gamma^{2}(G) \rightarrow G / \Gamma^{2-1}(G) .
$$

We argue by induction on $i$ that $G / \Gamma^{2}(G)$ is fgp. This is true by hypothesis if $i=1$, and the inductive step from $(i-1)$ to $i$ is just an application of Lemma 12 to the central extension above.

We complete the proof by taking $i$ sufficiently large that $\Gamma^{2}(G)$ is trivial.

${ }^{1}$ Our convention is that $\Gamma^{0} G=G, \Gamma^{2+1} G=\left[G, \Gamma^{2} G\right], i \geq 0$. 
We return now to the proof of Theorem 11 Suppose that $G$ is fgp Then $G_{a b}$ is fgp, so $G_{a b}^{\prime \prime}$ is fgp and, by Lemma $13, G^{\prime \prime}$ is fgp

Now let $p$ be an arbitrary prime and let $H$ be an fg subgroup of $G_{p}$ such that $H_{p}=G_{p}$ Then $H \cap G_{p}^{\prime}$, as a subgroup of an fg nilpotent group, is fg and $\left(H \cap G_{p}^{\prime}\right)_{p}=H_{p} \cap G_{p}^{\prime}=G_{p}^{\prime}$ This shows that $G^{\prime}$ is fgp

Finally, suppose $G^{\prime}$ and $G^{\prime \prime}$ both fgp We have the short exact sequence of abelian groups

$$
G^{\prime} / G^{\prime} \cap[G, G] \mapsto G_{a b} \rightarrow G_{a b}^{\prime \prime}
$$

and a surjection

$$
G_{a b}^{\prime} \rightarrow G^{\prime} / G^{\prime} \cap[G, G] .
$$

Since $G_{a b}^{\prime}$ is fgp, so is $G^{\prime} / G^{\prime} \cap[G, G]$. Since $G^{\prime} / G^{\prime} \cap[G, G]$ and $G_{a b}^{\prime \prime}$ are fgp, so is $G_{a b}$ We now apply Lemma 13 to complete the proof that $G$ is fgp

\section{THE CLASS OF FG-LIKE ABELIAN GROUPS}

We know that the subclass of the class of fgp nilpotent groups, which consists of fg-like groups, does not form a Serre class. The difficulty does not lie with the supplementary axioms, which continue to be valid, for the same reasons as before However, the basic axiom fails, even for fg-like abelian groups, since, as pointed out in the Introduction, a homomorphic image of an fg-like abelian group may fail to be fy-like However, insofar as abelian groups are concerned, this is the only part of the basic axiom which fails This is a consequence of the following theorem

THEOREM 2.1. Let $A$ be an abelian group Then $A$ is fg-like if and only if $A$ is fgp with $T A$ finite.

PROOF. Let $A$ be fg-like. Then $A$ is certainly fgp Moreover, if $A$ is $B$-like, with $B \mathrm{fg}$, then $T A \cong T B$, which is a finite group

Suppose conversely that $A$ is fgp with $T A$ finite. Then $F A$ is fgp, so that $F A_{p}$, for a fixed but arbitrary prime $p$, is the $p$-localization of a free abelian group $\mathbb{Z}^{k}$ of finite rank Moreover, $F A_{0}=\mathbb{Q}^{k}$, so that $k$ is independent of $p$. Thus $F A$ is $\mathbb{Z}^{k}$-like. Now $T A$ is finite. Thus, as in [CH1], $\operatorname{Ext}(F A, T A)=0$ and $A=T A \oplus F A \quad$ It follows that $A$ is $B$-like, where $B=T A \oplus \mathbb{Z}^{k}$

REMARK. The first, easier part of the argument, guaranteeing that an fg-like group is fgp with finite torsion subgroup, plainly extends to nilpotent groups

COROLLARY 2.2. Let $A^{\prime} \mapsto A \stackrel{k}{\rightarrow} A^{\prime \prime}$ be a short exact sequence of abelian groups. Then

i. $A^{\prime}$ is fg-like if $A$ is fg-like;

ii $A$ is fg-like if $A^{\prime}$ and $A^{\prime \prime}$ are fg-like.

PROOF. (i) Of course $A^{\prime}$ is fgp if $A$ if fgp. Moreover, $T A^{\prime}$ is a subgroup of $T A$ and hence is finite if $T A$ is finite. Thus (i) follows from Theorem 21

(ii) Of course $A$ is fgp if $A^{\prime}$ and $A^{\prime \prime}$ are fgp. Moreover $T A^{\prime} \mapsto T A \rightarrow k(T A)$ is a short exact sequence and $k(T A) \subseteq T A^{\prime \prime}$. Thus, since $T A^{\prime}$ and $T A^{\prime \prime}$ are finite, so is $T A$, and (ii) also follows from Theorem 2.1.

The rest of this paper is primarily motivated by our seeking to answer the question whether the analogue of Corollary 2.2 holds for nilpotent groups. We will see in the next section that it does hold for an important class of nilpotent groups, and in Section 4 that it does not hold in general

\section{ON NILPOTENT GROUPS WITH FINITE COMMUTATOR SUBGROUP}

We have proved elsewhere the following fundamental theorem [HM]:

THEOREM 3.1. Let $N \mapsto G \rightarrow Q$ be a short exact sequence of nilpotent groups, with $N$ finite Then $G$ is fg-like if and only if $Q$ is fg-like.

REMARK. We know, of course, that this theorem cannot be extended to the case in which $N$ is only assumed $\mathrm{fg}$. 
COROLLARY 3.2. Let $G$ be a nilpotent group with finite commutator subgroup Then $G$ is fglike if and only if $G_{a b}$ is fg-like

We now establish the analogues of the two parts of Corollary 22 for nilpotent groups with finite commutator subgroup

THEOREM 3.3. Let $G^{\prime}$ be a subgroup of a nilpotent group $G$ such that $[G, G]$ is finite Then $G^{\prime}$ is fg-like if $G$ is fg-like

PROOF. Since $G_{a b}$ is fg-like, so is its subgroup $G^{\prime} / G^{\prime} \cap[G, G]$ But $G^{\prime} \cap[G, G]$ is finite, so $G^{\prime}$ is fg-like by Theorem 31

REMARK. Obviously it suffices to assume $G_{a b}$ fg-like and $G^{\prime} \cap[G, G]$ finite

THEOREM 3.4. Let $G^{\prime} \mapsto G \rightarrow G^{\prime \prime}$ be a short exact sequence of nilpotent groups with $[G, G]$ finite Then if $G^{\prime}, G^{\prime \prime}$ are fg-like, so is $G$

PROOF. Consider the short exact sequence of abelian groups

$$
G^{\prime} / G^{\prime} \cap[G, G] \mapsto G_{a b} \rightarrow G_{a b}^{\prime \prime} .
$$

Since $G^{\prime} \cap[G, G]$ is finite, it follows from Theorem 31 that $G^{\prime} / G^{\prime} \cap[G, G]$ is fg-like Also $G_{a b}^{\prime \prime}$ is fglike, so, by Corollary 22 (ii), $G_{a b}$ is fg-like Thus, by Corollary $32, G$ is fg-like

It is not surprising, in the light of Theorems 33 and 34 that the analogue of Theorem 21 holds for nilpotent groups with finite commutator subgroup We first present an easy lemma

LEMMA 3.5. Let $G$ be nilpotent with $[G, G]$ a torsion group Then

$$
T\left(G_{a b}\right)=T G /[G, G] \text {. }
$$

PROOF. In the short exact sequence

$$
T G /[G, G] \mapsto G /[G, G] \rightarrow G / T G,
$$

the subgroup is torsion and the quotient is torsion free Thus $T G /[G, G]=T(G /[G, G])$

THEOREM 3.6. Let $G$ be nilpotent with $[G, G]$ finite Then, if $G$ is fgp with $T G$ finite, $G$ is fglike

PROOF. By Lemma $35 T\left(G_{a b}\right)$ is finite. Thus, since $G_{a b}$ is fgp, it follows from Theorem 21 that $G_{a b}$ is fg-like So, therefore, by Corollary 3.2 , is $G$.

Of course, Theorem 3.6 could be made the basis for alternative proofs of Theorems 3.3, 3.4

\section{A COUNTEREXAMPLE}

In this section we construct an example of an fgp nilpotent group $G$ which is not fg-like but which may be obtained as an extension of an fg-like group by an fg-like group We then embed $G$ in an fg-like group $H$. Thus we have a counterexample to the generalization of each part of Corollary 22 to nilpotent groups. The group $H$ (and hence also the group $G$ ) is torsion free and nilpotent of class 2 Thus $G$ also provides a counterexample to the generalization of Theorem 21 to nilpotent groups. Of course, if such a generalization had been valid, we could have generalized Corollary 22 also

Let $A \subseteq \mathbb{Q}$ be the group of pseudo-integers [H1] generated by the rationals $\frac{1}{p}$, as $p$ varies over all the primes (see the Introduction). Let $C=\langle\xi\rangle$ be a cyclic infinite group acting on $A \oplus \mathbb{Z}$ by the rule

$$
\xi \cdot(a, n)=(a+n, n),
$$

and let $G$ be the semidirect product for this action. Since $A \oplus \mathbb{Z}$ and $C$ are torsion free, $G$ is torsion free, and $G$ is plainly an extension of an fg-like group by an fg-like group If we write $A \oplus \mathbb{Z}$ multiplicatively, then $G$ has the presentation (with $\tau_{p}$ 'representing' $\frac{1}{p}$ )

$$
G=\left\langle\tau_{p}, \alpha, \xi \mid \tau_{p} \tau_{q}=\tau_{q} \tau_{p}, \tau_{p} \alpha=\alpha \tau_{p}, \tau_{p} \xi=\xi \tau_{p}, \tau_{p}^{p}=\tau_{q}^{q}, \xi \alpha \xi^{-1}=\tau \alpha, \forall p, q\right\rangle,
$$

where

$$
\tau=\tau_{p}^{p}, \forall p
$$


It is plain from (42) that $[G, G]=\langle\tau\rangle$ and that $\tau_{p} \in Z G$ Thus $[G, G] \subseteq Z G$ so that $G$ is nilpotent of class 2 Further we see from (4 2) that

$$
G_{a b}=G /\langle\tau\rangle=\left\langle\bar{\tau}_{p}, \bar{\alpha}, \bar{\xi} \mid \bar{\tau}_{p}^{p}=1, \forall p\right\rangle
$$

Thus $G_{a b}$ has $p$-torsion for all $p$, so $G_{a b}$ is not fg-like Neither, then, is $G$, so that $G$ is an example of an fgp nilpotent group of class 2 which is an extension of an fg-like group by an fg-like group, but which is not itself fg-like

We now embed $G$ in $H$ by adding to (4 2) new generators $\xi_{p}$, for all $p$, and new relations

$$
\xi_{p} \xi_{q}=\xi_{q} \xi_{p}, \xi_{p} \tau_{q}=\tau_{q} \xi_{p}, \xi_{p} \alpha \xi_{p}^{-1}=\tau_{p} \alpha, \xi_{p}^{p}=\xi, \forall p, q .
$$

Plainly $H$ may be regarded as a semidirect product for an action of $A$ on $A \oplus \mathbb{Z}$ and is therefore also torsion free We now have $[H, H]=\left\langle\tau_{p}, \forall p\right\rangle$ and $Z H=\left\langle\tau_{p}, \forall p\right\rangle$, so $H$ is nilpotent of class 2

We now fix an arbitrary prime $p$ and consider the $p$-localization $H_{p}$ of $H$. The $p$-localization of $A \oplus \mathbb{Z}$ is $A_{p} \oplus \mathbb{Z}_{p}$, where $A_{p}$ is the cyclic $\mathbb{Z}_{p}$-module generated by $\frac{1}{p}$. Thus, again writing multiplicatively, with $\tau_{p}$ representing $\frac{1}{p}$,

$$
H_{p}=\left\langle\tau_{p}, \alpha_{p}, \xi_{p} \mid \tau_{p} \alpha_{p}=\alpha_{p} \tau_{p}, \tau_{p} \xi_{p}=\xi_{p} \tau_{p}, \xi_{p} \alpha_{p} \xi_{p}^{-1}=\tau_{p} \alpha_{p}\right\rangle_{p}
$$

where \langle\rangle$_{p}$ means that the terms inside the braces provide a presentation of $H_{p}$ as $p$-local group Let $K$ be the group given by the presentation

$$
K=\left\langle\tau, \alpha, \xi \mid \tau \alpha=\alpha \tau, \tau \xi=\xi \tau, \xi \alpha \xi^{-1}=\tau \alpha\right\rangle .
$$

It is then plain that $K$ is an fg nilpotent group of class 2, and comparison of (4 6), (4 7) shows that

$$
K_{p} \cong H_{p}, \forall p \text {. }
$$

Thus $H$ is $K$-like, so that $H$ is an fg-like torsion free nilpotent group of class 2 with a subgroup $G$ which is not fg-like. Notice that $K$ is just the free nilpotent group of class 2 on 2 generators, $K=F_{2}\langle\alpha, \xi\rangle$

Of course, our construction could be imitated with $A$ replaced by any other group of pseudo-integers not isomorphic to $\mathbb{Z}$ If $A=\left\langle\frac{1}{p^{m(p)}}, \forall p\right\rangle$, then $A / \mathbb{Z}=\underset{p}{\oplus} \mathbb{Z} / p^{m(p)}$. But the analogue of (4 4) tells us that the torsion subgroup of $G_{a b}$ is precisely $\underset{p}{\oplus} \mathbb{Z} / p^{m(p)}$, so that, as we vary $A$, we vary the isomorphism class of $G$. Since there is a continuum of groups of pseudo-integers [H1], there is a continuum of groups $G$ which can be constructed in this way having the properties attributed above to our special choice of $G$. In particular each such group $G$ may be embedded in a suitable $K$-like nilpotent group $H$, where $K$ is the free nilpotent group of class 2 on 2 generators

\section{REFERENCES}

[CH1] Casacuberta, Carles and Peter Hilton, On the extended genus of finitely generated abelian groups, Bull. Soc. Mat. Belg. (Series A), XLI, 1 (1989), 51-72.

[CH2] Casacuberta, Carles and Peter Hilton, On special nilpotent groups, Bull Soc. Mat. Belg. (Series B), XLI3 (1989), 261-273.

[CH3] Casacuberta, Carles and Peter Hilton, On nilpotent groups which are finitely generated at every prime, Expositiones Mathematicae, 10 (1992), 385-402.

[H1] Hilton, Peter, On groups of pseudo-integers, Acta. Math. Sinica 4, 2 (1988), 189-192.

[H2] Hilton, Peter, On the extended genus, Acta Math. Sinica 4, 4 (1988), 372-382

[H3] Hilton, Peter, On a family of Serre classes of nilpotent groups, Journ. Pure and App. Alg. 89 (1993), 127-133.

[HM] Hilton, Peter and Robert Militello, Some remarks on almost finitely generated nilpotent groups, Publicacions Matemàtiques, 36 (1992), 655-662.

[HR] Hilton, Peter and Joseph Roitberg, Generalized $C$-theory and torsion phenomena in nilpotent spaces, Houston J. of Math. 2 (1976), 525-559.

[M1] Militello, Robert, On Cayley-Hamilton Properties in Certain Classes of Groups, SUNY Binghamton (1991).

[M2] Mislin, Guido, Nilpotent groups with finite commutator subgroups, Lecture Notes in Math 418, Springer Verlag (1974), 103-120. 


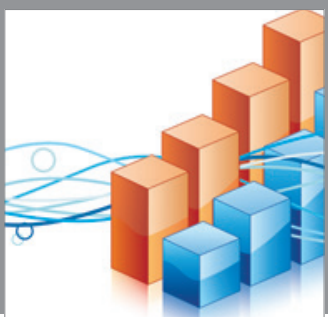

Advances in

Operations Research

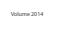

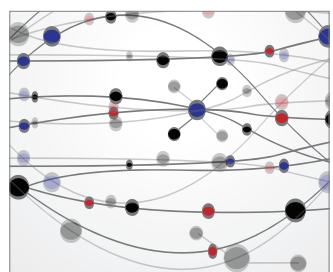

\section{The Scientific} World Journal
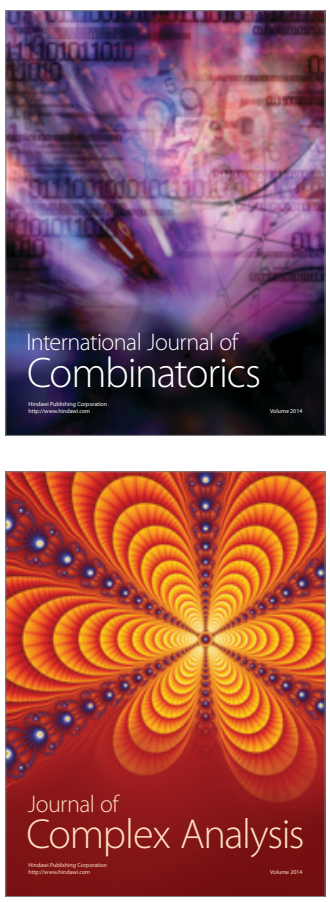

International Journal of

Mathematics and

Mathematical

Sciences
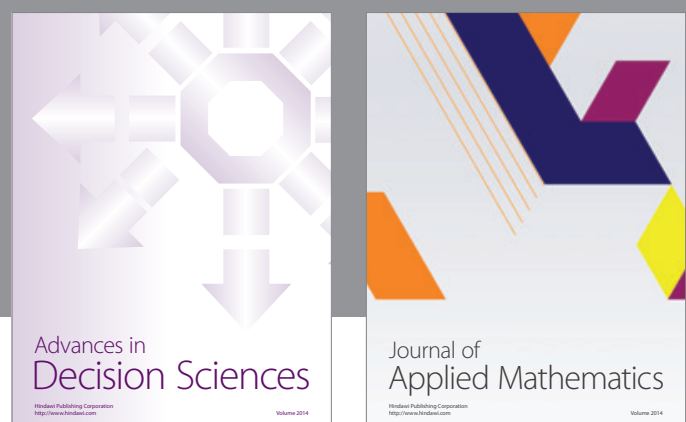

Journal of

Applied Mathematics
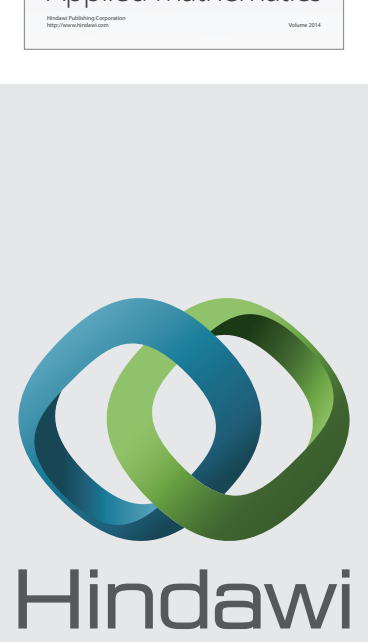

Submit your manuscripts at http://www.hindawi.com
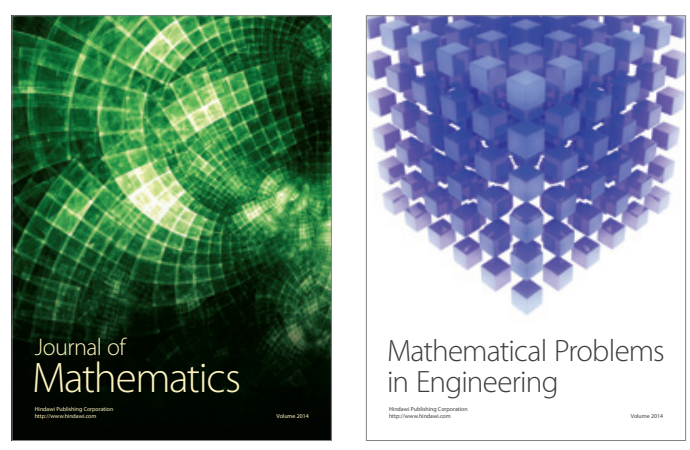

Mathematical Problems in Engineering
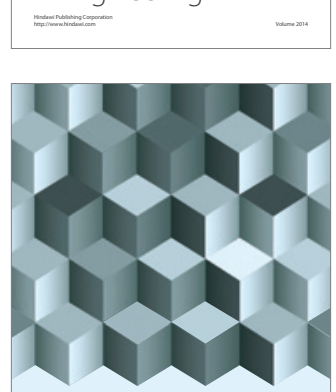

Journal of

Function Spaces
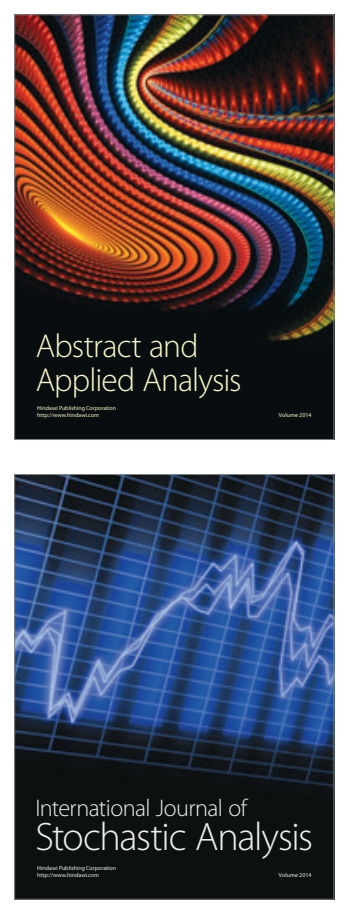

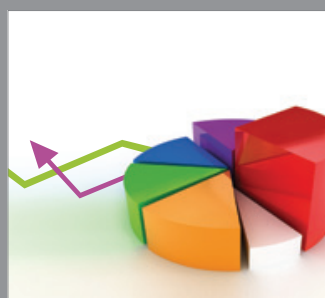

ournal of

Probability and Statistics

Promensencen
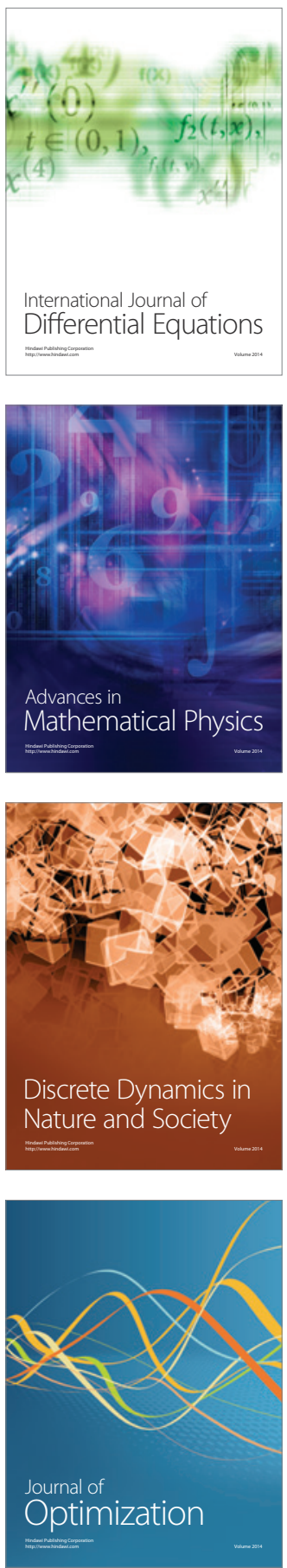